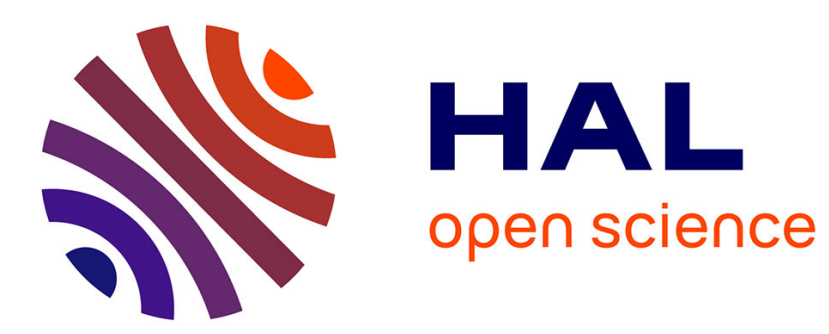

\title{
Flexible Multipoint-to-Multipoint Routing Protocol in Ultra-Dense Nanonetworks
}

\author{
Lina Aliouat, Hakim Mabed, Julien Bourgeois
}

\section{To cite this version:}

Lina Aliouat, Hakim Mabed, Julien Bourgeois. Flexible Multipoint-to-Multipoint Routing Protocol in Ultra-Dense Nanonetworks. International Symposium on Mobility Management and Wireless Access, Nov 2019, Miami Beach, FL, United States. 10.1145/3345770.3356746 . hal-02392510

\section{HAL Id: hal-02392510 https://hal.science/hal-02392510}

Submitted on 4 Dec 2019

HAL is a multi-disciplinary open access archive for the deposit and dissemination of scientific research documents, whether they are published or not. The documents may come from teaching and research institutions in France or abroad, or from public or private research centers.
L'archive ouverte pluridisciplinaire HAL, est destinée au dépôt et à la diffusion de documents scientifiques de niveau recherche, publiés ou non, émanant des établissements d'enseignement et de recherche français ou étrangers, des laboratoires publics ou privés. 
archives-ouvertes

\title{
Flexible Multipoint-to-Multipoint Routing Protocol in Ultra-Dense Nanonetworks
}

\author{
Lina Aliouat, Hakim Mabed, Julien Bourgeois
}

\section{To cite this version:}

Lina Aliouat, Hakim Mabed, Julien Bourgeois. Flexible Multipoint-to-Multipoint Routing Protocol in Ultra-Dense Nanonetworks. International Symposium on Mobility Management and Wireless Access, Nov 2019, Miami Beach, FL, United States. hal-02392510

\section{HAL Id: hal-02392510 \\ https://hal.archives-ouvertes.fr/hal-02392510}

Submitted on 4 Dec 2019

HAL is a multi-disciplinary open access archive for the deposit and dissemination of scientific research documents, whether they are published or not. The documents may come from teaching and research institutions in France or abroad, or from public or private research centers.
L'archive ouverte pluridisciplinaire HAL, est destinée au dépôt et à la diffusion de documents scientifiques de niveau recherche, publiés ou non, émanant des établissements d'enseignement et de recherche français ou étrangers, des laboratoires publics ou privés. 


\section{Flexible Multipoint-to-Multipoint Routing Protocol in Ultra-Dense Nanonetworks}

\author{
Lina Aliouat \\ lina.aliouat@univ-fcomte.fr \\ Univ. Bourgogne Franche-Comté \\ Montbéliard, France
}

\author{
Hakim Mabed ${ }^{*}$ \\ hmabed@femto-st.fr \\ Univ. Bourgogne Franche-Comté \\ Montbéliard, France
}

\author{
Julien Bourgeois* \\ Univ. Bourgogne Franche-Comté \\ Montbéliard, France \\ julien.bourgeois@femto-st.fr
}

\begin{abstract}
New applications in the field of radio networks require a high concentration of micro-machines (micro-robots, sensors/actuators) in a small space. Those devices are characterized by a high volatility and limited computing, storage and energy capabilities. Traditional routing approaches in ad hoc networks are unusable due to a significant amount of additional control traffic and a lack of robustness regarding the instability of the nodes. In this paper, we present an original, efficient and intuitive distributed routing protocol in ultra-dense terahertz networks, called Multipoint-to-Multipoint Routing Protocol (M2MRP), which is an emanation of electrostatic physics.

A complexity analysis is performed to compare the M2MRP protocol with classical methods. Our study shows that the proposed protocol takes advantage of the nodes density to define a robust routing policy with a moderate additional traffic control. In addition, routing paths are adapted gradually and continuously according to the nodes location (mobility), availability (failures), congestion and energy level.

Simulations show that the M2MRP routing protocol significantly outperforms the well-known routing protocols for dense networks both in terms of the number of exchanged messages and of success rate, making this routing protocol the most suitable for systems such as swarm micro-robots, programmable matter and ultra-dense sensor networks.
\end{abstract}

\section{KEYWORDS}

Terahertz nanonetwork, dense network, routing protocol, multipointto-multipoint routing

\section{ACM Reference Format:}

Lina Aliouat, Hakim Mabed, and Julien Bourgeois. 2019. Flexible Multipointto-Multipoint Routing Protocol in Ultra-Dense Nanonetworks. In 17th ACM International Symposium on Mobility Management and Wireless Access (MobiWac '19), November 25-29, 2019, Miami Beach, FL, USA. ACM, New York, NY, USA, 7 pages. https://doi.org/10.1145/3345770.3356746

\footnotetext{
Permission to make digital or hard copies of all or part of this work for personal or classroom use is granted without fee provided that copies are not made or distributed for profit or commercial advantage and that copies bear this notice and the full citation on the first page. Copyrights for components of this work owned by others than ACM must be honored. Abstracting with credit is permitted. To copy otherwise, or republish, to post on servers or to redistribute to lists, requires prior specific permission and/or a fee. Request permissions from permissions@acm.org. MobiWac '19, November 25-29, 2019, Miami Beach, FL, USA

ACM ISBN 978-1-4503-6905-3/19/11 .\$15.00

https://doi.org/10.1145/3345770.3356746
}

\section{INTRODUCTION}

During the last few years, there have been important advances in the field of radio devices manufacturing, both in terms of miniaturization (Micro-electro-mechanical systems (MEMS) and antennas) and cost reduction. These advances have opened the way to the design of systems where hundreds of thousands of radio nodes can co-exist in a reduced space. Foreseen applications include robotics swarms, programmable matter [1], massive multi-core processing [2], Wireless NanoSensor Network (WNSN) [3] and Wireless Body Access Network (WBAN) [4]. In such systems, network densification helps increasing the accuracy of extracted information and efficiently covering an area.

Due to the limited energy capacity of the nodes and their high density, the radio range of each node is reduced. A communication between two distant nodes is then relayed by intermediate nodes. The routing problem in ad hoc network is widely covered by the literature. Mainly, the classical routing approaches could be classified into proactive and reactive protocols [5]. In proactive protocols (OLSR [6], Babel [7], DREAM [8] and DSDV [9] ), each node computes and stores in advance the optimal paths or just the next node in this path, towards one, several or all nodes. These approaches suffer of lack of responsiveness in case of nodes failure requiring the path recalculation. In reactive protocols, such as AODV [10], ABR[? ] and DSR[? ], the path followed by the communication data is computed in real-time by exchanging route requests (flooding mechanism), which leads to additional latency and additional control traffic. The flooding impact is accentuated by the high density of the network.

Recent few works on routing use the analogy between dense wireless network and physical phenomena such as the interaction of charged particles in electrostatics [11] or molecules interaction in fluid substance [12]. Major of these works model the problem as a global optimization problem and assume the presence of a central unit with a global knowledge on the network state. Wei et al. [12] present a distributed routing protocol based of fluid dynamics. However, the protocol assumes that each node knows its coordinates and is able to determine the relative positions and distances of its neighbors.

In all the works cited above, a path or a route designates a list of nodes that defines a succession of Point-to-Point links. Due to the node volatility in ultra dense nanonetwork context, proactive point-to-point based routes are irrelevant, which means that only reactive approaches could be considered with point-to-point based routes.

New routing paradigm was proposed in [13] and [14]. These approaches are based on multipoint-to-multipoint paths and offer a more reliable connection especially under the high volatility context 


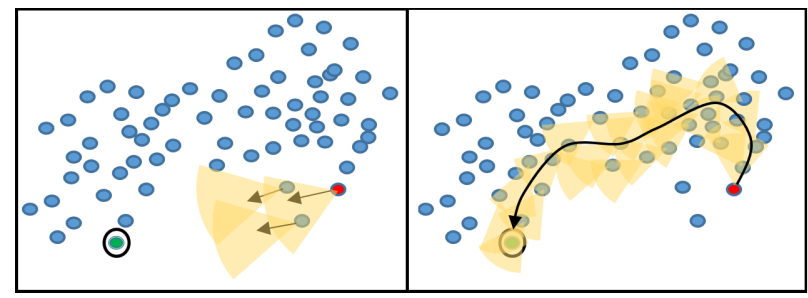

Figure 1: Comparison between direct line multipoint-tomultipoint approach (left) and M2MRP protocol (right). The surrounded node is a sink and the dark one is the source. The direct line approach fails to route the message until the sink node, whereas M2MRP approach succeeds.

of nanonetworks. The data are transmitted from the source node to the destination node by crossing successive areas. At each hop, all the nodes of the current area re-transmit the packet to the next area and so on until the packet reaches the area containing the destination node. The successive areas passed through by the packet correspond to those located on the direct line between the source and the destination nodes. Therefore the protocol is not adapted to the case where the network nodes form a non-convex shape (see Figure 1).

\section{CONTRIBUTIONS}

In this paper, we propose a proactive multipoint-to-multipoint routing protocol (M2MRP) for ultra-dense nanonetworks with volatile nodes. The density of the network is exploited in order to increase the communication reliability. Packet routing follows a sequence of multi-point to multi-point links (see Figure 1), which offers a better protection against packet lost.

Unlike direct line routing protocols [14], the M2MRP uses adaptation mechanisms that allow to continuously adjust the circulation flows of data according to network deployment (mobility), nodes availability (curative adaptation) and energy state (preventive adaptation). This way, the adequacy of a node to serve as a relay point varies over time according to its energy state and the state of its neighboring nodes.

Additionally, unlike other methods, M2MRP uses the Terahertz beam steering technology [15] to improve the spatial coverage of the nodes (the node radiates in a specific direction corresponding to the targeted reception nodes) leading to a more efficient use of energy, a better interference control and a reduction of short multiple reception loops (reception of the same packet several times). M2MRP allows every node to determine the best reception and transmission directions that optimize the circulation flow of traffic from any node to the sink nodes. Therefore, the flooding effect and the cumulative interferences are significantly reduced.

Every original received packet (received from a node situated in the reception direction) is just sent in the transmission direction without checking the sender or the destination identity. Finally, M2MRP protocol leads to an efficient use of wake-up receiver technology [16], since the energy generated by transmitters is steered in one direction to activate only a part of the physical neighbors corresponding to the next hop nodes. This procedure provides a low-latency communication environment when nodes are activated just in time to receive a packet and to re-transmit it.

\section{ROUTING PROBLEM DESCRIPTION}

\subsection{Multiple sinks problem}

Routing problem in ad hoc networks with multiple sources and multiple destinations is a very frequent problem $[17,18]$. This problem is particularly relevant in wireless nanosensor networks and nanonetworks. We consider here the case where source nodes can be any node in the network and sink nodes are particular nodes acting as gateways to the data processing unit. Sink nodes are particular because they are assumed not to be impacted by the volatility phenomena that affects ordinary nodes.

We assume that the ad hoc nanonetworks are sufficiently dense to allow an intensive coverage of the deployment area. This density is due to the miniaturization technologies such as graphene antenna [19] and MEMS components [20]. Terahertz band is then envisaged as an adequate radio environment to support communications between sub-millimeter devices.

\subsection{Access protocol in terahertz nanonetwork}

TS-OOK/PHLAME [21] is one of the most promising Terahertz access protocol. TS-OOK/PHLAME is a pulse-based modulation protocol where every communication is encoded by a sequence of $0 / 1$ symbols transmitted at regular intervals called Time symbol. The 1 symbol is sent as a electromagnetic pulse of a duration $T p$ (arround 100 femtosec) while a 0 symbol corresponds to a silence. The symbol rate (interval between two consecutive symbols) is notified by the sender during the communication announcement over the control channel.

The control channel is a carrier-less logical channel associated with the constant symbol rate $T s_{0}$. The receiver node listens to the control channel and extracts any sequence of symbols with a $T s_{0}$ interval, in order to detect an announcement packet with an indication about the symbol rate of the data communication, $T s$. Listening to the control channel costs in terms of energy and memory because detecting an announcement requires the storage and the processing of the received symbols. Therefore, the use of wake-up receivers [16] is necessary in order to optimize the announcement detection procedure. A wake-up receiver is a radio technology providing a radio-on-demand connection where the nodes are activated just in time to receive the data.

\subsection{The Multipoint-to-Multipoint Routing Protocol (M2MRP)}

Under a context of high nodes volatility, the proactive point-topoint model is unreliable, i.e. with a high packet lost rate. While reactive protocols based on flooding processes lead to a high redundancy, interference and high energy consumption level as well as a high latency. The idea of the M2MRP protocol is to build a data circulation pattern that defines the paths followed by the date from any ordinary node to one of the sink nodes (see Figure 2). The circulation pattern of the traffic is modeled with a distributed data structure that locally associates with every ordinary node, 
two steering directions one for receiving and another one for sending data. At this stage of the work, we simply consider that the reception and transmission directions are opposite. To compute

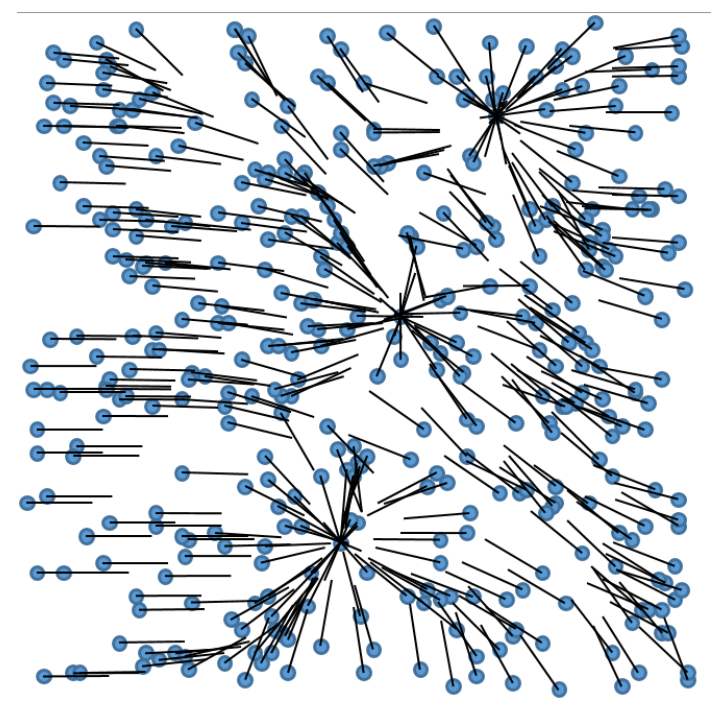

Figure 2: Data circulation pattern. Only the main transmission directions are depicted. The reception direction is either omni-directional or the opposite of the transmission direction.

these lines of circulation flow, we were inspired by the electrostatic physics to determine how the nodes compute the best transmission direction (and not the best next hop node) to reach one of the sink nodes. Three main principles govern the behavior of the M2MRP protocol:

- the attractiveness effect: every node attracts all nodes within a given range.

- the distance effect: the attraction level of a given node $n$ on another one is proportional to the received signal power from the node $n$.

- the attractiveness vector of a given node is the result of the attractiveness vectors of its direct neighbors and corresponds to the transmission direction of the node (i.e. the node's antenna radiates in this direction).

The third principal means that the attractiveness vector of a given node is computed starting from the attractiveness vectors of its direct neighbors. Therefore, the attractiveness of each node impacts the attractiveness of farther nodes by the effect of intermediate nodes. The distributed propagation process leads to the formation of the circulation flow lines defining the data circulation paths from ordinary nodes to the sink nodes.

More formally, let $N$ be a nanonetwork composed of a set of ordinary nodes, $O$ and a set of sink nodes, $S$. Each node involves a directional antenna that can be steered in a direction $d \in D$ with a fixed opening angle $\alpha$. The M2MRP protocol proceeds by periodic updates of the circulation flows to reflect the evolution of nodes state: availability and energy level. In asynchronous way, each node proceeds per cycles (See Figure 3) called routing cycle. The routing cycle duration is $10^{12} \times T p=100 \mathrm{~ms}$ and is subdivided into 1000 data cycles of a duration $10^{9} \times T p=0.1 \mathrm{~ms}$. The last data cycle is dedicated to the routing update procedure and forms the listening cycle. During the listening cycle, each ordinary node, $o \in O$, captures the control packets sent by the neighboring nodes (the antenna is used in omnidirectional mode). The control packets are particular packets with a specific signature and contain the attractiveness vector of the transmitter node and its energy level. The signal power of the received control packets and the attractiveness vectors of all neighbors are processed in order to determine the main sending direction, $\overrightarrow{d(o)}$, of the node $o$.

Ordinary communication cycles are subdivided into 1000 data packet cycles. The $1000-|D|$ first packets are used to eventually send data packets in the main direction $\overrightarrow{d(o)}$ of the node. During the last $|D|$ data packet cycles, the node sends $|D|$ control packets each one in a different direction $d \in D$. Therefore, during the listening cycle, the node is ensured to detect all the control packets of its available neighbors in all directions.

Every $100 \mathrm{~ms}$, an ordinary node $o$ updates its attractiveness vector using the received control packets during the listening cycle $(0.1 \mathrm{~ms})$. Let $V(o)$ the set of neighboring nodes of $o$. We notice pow $(v, o)$ the power of the received signal from the node $v$ at the node $o$. If $o$ receives different control packets from the same neighbor $v$ with different antenna directions, the strongest signal is then selected.

$$
\operatorname{pow}(v, o)=\max _{\vec{d} \in D} \operatorname{pow}(v, \vec{d}, o)
$$

Let $\overrightarrow{d(v)}$ be the attractiveness vector of the node $v$ sent in the last control packet of the node $v$.

The attractiveness vector of the node $o$ can then be updated according to the expression 2. It should be noted that attractiveness vectors are normalized.

$$
\begin{aligned}
& \overrightarrow{d(o)}=\sum_{v \in V(o)} \overrightarrow{d(v)} * \operatorname{pow}(v, o) \\
& \overrightarrow{d(o)}=\overrightarrow{d(o)} /\|\overrightarrow{d(o)}\|
\end{aligned}
$$

However, equation 2 presents an important drawback because the weighted sum of neighbors attractiveness vectors may lead to an attractiveness vector oriented to low dense or even empty area as shown in the figure 4 . The transmitted data sent from the node may not reach the sink nodes.

To overcome this problem, we propose another expression of the attractiveness computation, where the transmission direction or attractiveness vector, $\overrightarrow{d(o)}$, is computed by each node $o$ as follow:

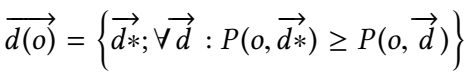

$$
\begin{aligned}
& \text { with : } \\
& P(o, \vec{d})=\sum^{v \in V(o)} \operatorname{pow}(v, o) \times \cos (\vec{d}, \overrightarrow{d(v)}) \times E(v)
\end{aligned}
$$

Where $E(v)$ designates the current energy level of the neighboring node $v$ and $\vec{d}$ corresponds to the normalized vector which the direction $d$ belongs to the set $D$.

The equation 3 guarantees that the resulting direction computed by the node $o$ is the best regarding the concentration of the next hop nodes and their distance from the node $o$. The introduction of the energy level of the nodes gives a natural and efficient way 


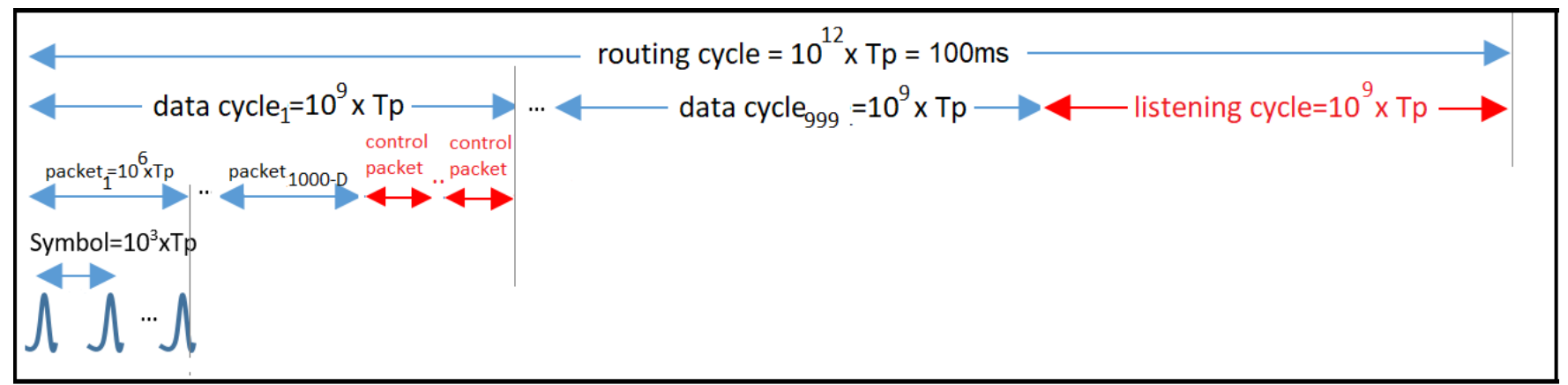

Figure 3: Routing cycle: every $100 \mathrm{~ms}$, the node recomputes its attractiveness vector (the transmission direction).

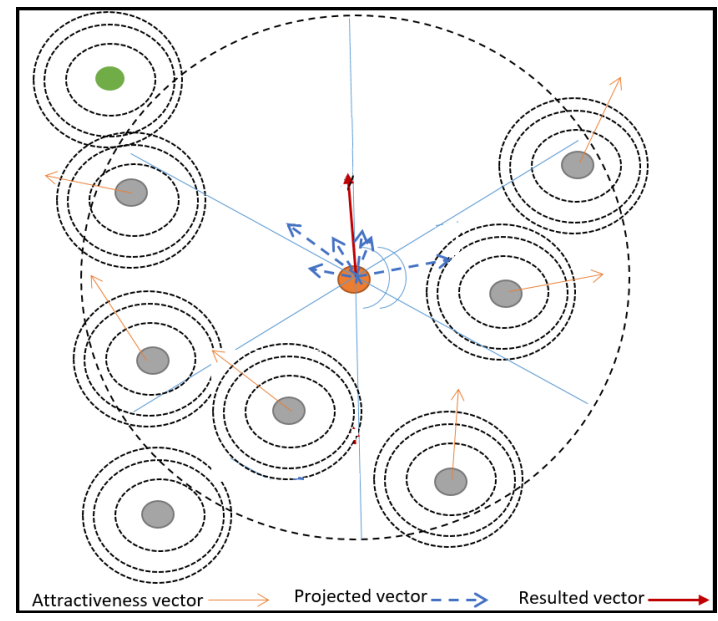

Figure 4: Attractiveness vector obtained with equation 2: the reference node is surrounded by 6 neighbors with a sufficiently strong signal. The reference node computes the weighted sum (regarding the signal power) of the neighbors attractiveness vectors. In the example, the obtained vector is then badly oriented.

to dynamically adapt the computed paths (i.e. flows circulation) according to the nodes capacities. In other words, the overused areas become less attractive, allowing an implicit load balancing scheme.

Sink nodes, $s \in S$, present a specific case where the sent attractiveness vector $\overrightarrow{d(s)}$ is $\vec{\infty}$. When an ordinary node o receives one or several control packets from sinks ( $\vec{\infty}$ vector), it sets its own attractiveness vector, $\overrightarrow{d(o)}$, in the direction of the sink node (current direction $\vec{d} \in D$ ) and ignores the result of the equation 3 .

Algorithm ?? describes more formally the overall routing protocol.

\section{COMPLEXITY STUDY}

In this section, we analyze a set of the major routing methods used in the literature. For this purpose, many criteria are considered:

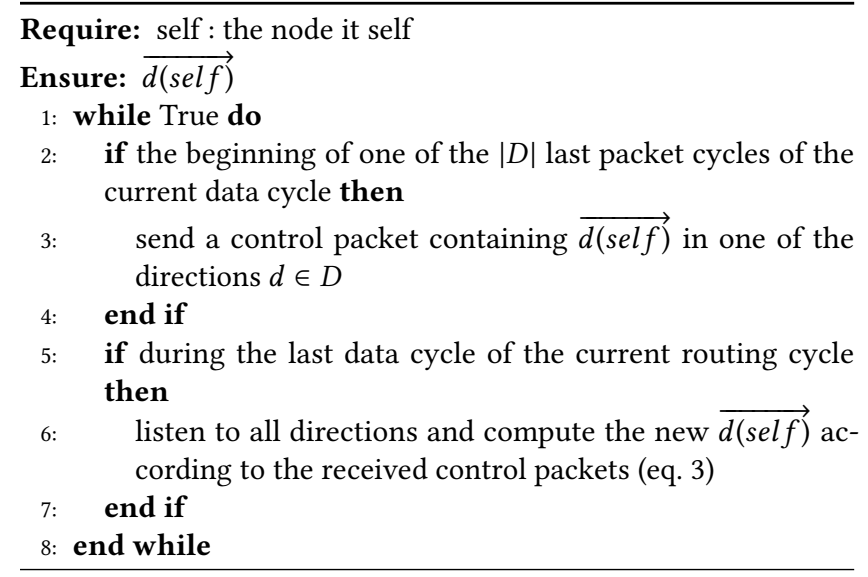

- The spatial complexity of the protocol corresponds to the amount of stored data necessary for the protocol operation.

- The routing efficiency corresponds to the time needed for transmitting the data from the source node to one of the sink nodes. This criteria allows to appreciate the quality of the routing path.

- The communication complexity corresponds to the number of sent messages required to broadcast one message from a source node. This number gives an idea about the global amount of energy consumed.

- Number of received messages that corresponds to the number of received messages by all nodes but not necessarily resent. The number of received messages impacts on the amount of generated interference.

- Number of control messages corresponds to the number of exchanged messages before selecting the routing path.

- The need for checking the source and the final destination nodes of the received messages allows to appreciate the additional delay and energy consumption required by the protocol.

In Table 1, we give a comparison of the 5 studied methods according to the 7 criteria listed above. From the table, we deduce that the OLSR approach is very demanding for memory capacity $\left(|V|^{2}\right)$, where $|V|$ is the average number of neighbors. On the contrary, AODV presents a good memory complexity $(|S|$ where $S$ is the 
set of sinks) but since AODV is a reactive approach (on demand), the construction of the optimal path could take time (up to $|E|$ messages where $|E|$ is the number of pairs of neighbor nodes). In addition, AODV is a point-to-point approach, which represents a low reliability condition.

The naive broadcast approach guarantees the fastest transmission of data to the sink nodes but the number of sent and received messages is significant and leads to a high amount of energy consumption and a high interference level. The multi-relay approaches (M2MRP and SLR) generate less exchanged messages than the flooding methods. However, SLR method uses the coordinates of the source and sink nodes to determine the relay nodes (nodes in the zones crossed by the direct line between the source and destination nodes). Consequently, the efficiency of SLR approach depends on the average density of a zone $Z$ (average number of nodes in one zone) and the geographical distance between the source and the destination nodes, $r$. Dense zones can lead to high level of exchanged messages while blank zones can lead to communication failures (see Figure 1, left part). Finally, M2MRP protocol is the only protocol that does not check the source of the message nor its final destination before relaying it, which represents a gain of time and energy.

M2MRP protocol is a distributed, robust and scalable protocol with a constant memory complexity on each node. Indeed, the attractiveness vector of $o, \overrightarrow{d(o)}$, is obtained by computing, on each direction $d \in D$, the sum of the projections of neighboring attractiveness vectors and then to select the best direction (See Equation 3). Therefore, there is no need to store the attractiveness vectors of all the neighbors before computing the attractiveness vector. The time complexity for the calculation of the attractiveness vector of a given node is linearly correlated to the number of its neighboring nodes. In ultra dense nanonetworks, it is possible to vary the radio coverage range in order to adapt the number of considered neighbors. The amount of data diffused by each node at every data cycle $(0.1 \mathrm{~ms})$ is too small making possible the continuous updating of the attractiveness vectors. Furthermore, the bad reception of the control packet of a given neighbor does not have enormous impact on the computed attractiveness vector.

\section{TESTS AND RESULTS}

\subsection{Simulations and scenarios}

For our experiments, we only compared the methods that meet the reliability criteria, thus node-to-node routing are ignored. In addition and regarding to its spatial complexity, the standard OLSR approach is considered not suitable for ultra dense nanonetworks. Therefore, three multiple relays to multiple relay protocols are compared: naive broadcast method, SLR and M2MRP. We run each protocol over 14 scenarios depicting two network topologies: the square topology and the bow tie topology (see Figure 5). In the square topology, the nodes of the network are uniformly distributed, while in bow tie topology the network forms a non convex shape similar to a bow tie.

All simulations are made by assuming a deployment zone of $100 \mathrm{~cm} \times 100 \mathrm{~cm}$ and a radio coverage range of $20 \mathrm{~cm}$. We tested two versions of the M2MRP protocol using two different antenna radiation angles: $60^{\circ}$ and $120^{\circ}$ while the reception angle is fixed to $120^{\circ}$

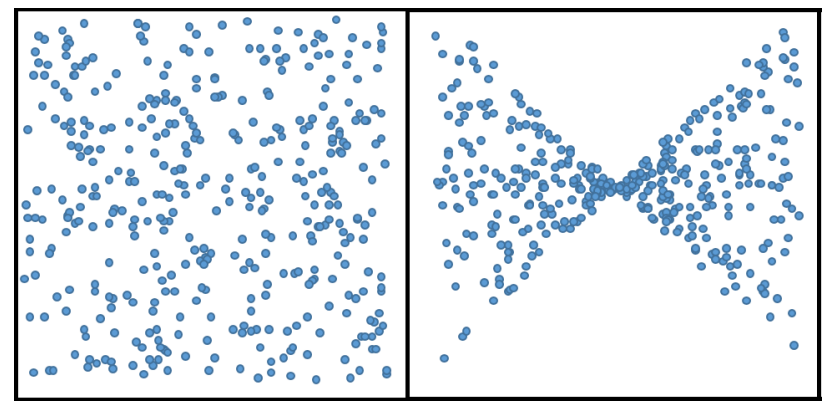

Figure 5: The two studied scenario topologies.

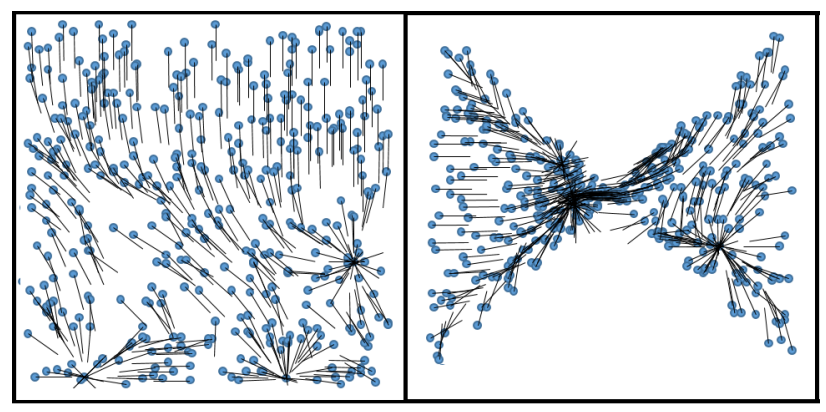

Figure 6: Data circulation patterns obtained for two scenarios with two different topologies.

and is at the opposite direction of the transmission direction defined by the attractiveness vector. The received power is computed as a function of the distance between the sender and the receiver nodes and decreases as the square of the distance between the nodes.

\subsection{Visual results of M2MRP}

In Figure 6, we display an example of the circulation flows produced by the M2MRP protocol on two different scenarios with two different topologies. Figure 6 shows that the data circulation flows fit well with nodes distribution. In particular, in the bow tie topology, the routes use the junction area to connect the left and the right parts of the network.

\subsection{Energy level and congestion processing}

As shown in equation 3, the energy level is a determinant factor of the attractiveness of a given node. When an area of the network is overloaded, energy level of its nodes becomes lower. Equation 3 provides a solution to reduce the congestion over the concerned areas. The effect of energy level on the construction of the circulation flows is given in Figure 7. On the left, the shadowed square represents nodes where the energy level is much lower than the remaining network. On the right, the shadowed square contains nodes with ordinary energy level. We observe that, on the left, the circulation flows avoid the square area and route data traffic differently toward the sink nodes.

The adaptation of the distributed routing protocol to the nodes state evolution is of course not instantaneous but the propagation of the local information remains fairly fast due to the frequency of 


\begin{tabular}{|c|c|c|c|c|c|c|c|}
\hline Method & $\mathrm{O}(\mathrm{mem})$ & $\mathrm{O}(\mathrm{t})$ & \#sent mess & \#rcv mess & \#ctrl mess & check src & check dst \\
\hline M2MRP & $\mathrm{O}(1)$ & Denser Path & $\ll N$ & $\ll|V| \times N$ & 0 & & \\
Flooding & $\mathrm{O}(1)$ & Shortest Path & $|D| \times|N|-|S|$ & $|V| \times N$ & 0 & $*$ & \\
SLR & $0(1)$ & Direct Path & $Z \times r$ & $Z \times 8 \times r$ & 0 & & $*$ \\
AODV & $|S|$ & Shortest Path & $r$ & $r$ & upto $|E|$ & & $*$ \\
OLSR & $|V|^{2}$ & $>$ shortest path & $<|D| \times N$ & $<|V| \times N$ & 0 & $*$ & $*$ \\
\hline
\end{tabular}

Table 1: Complexity comparison of 5 major routing approaches. $|N|$ is the number of nodes, $|S|$ is the number of sinks, $|D|$ is the number of antenna directions, $r$ is the number of zones between the source and the destination nodes, $Z$ is the size of a zone and $|E|$ is the number of pairs of neighbors.

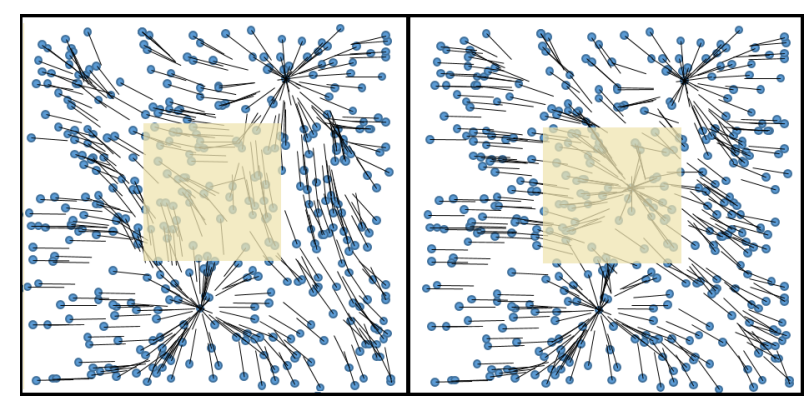

Figure 7: Energy effect: The yellow square is an area with low energy nodes (on the left). The introduction of the energy allows to divert data flows from this area.

local updates (every 100ms). For instance, the distance of any two nodes is less than 10 hops, the attractiveness vector of a given node takes into account all the updates (energy and availability changes) occurred on the network in the last second.

\subsection{Performance analysis}

To assess the efficiency of Multipoint-to-Multipoint Routing Protocol (M2MRP), several densities of network are tested: 100, 200, 300, 400, 500, 750 and 1000 nodes. For each topology, the scenario with $N$ nodes is composed of the same nodes (same coordinates) than the scenario with $M(M<N)$ nodes and $N-M$ additional nodes. In addition, the simulated traffic (source of packets) is the same over all scenarios with the same topology. This way, we guarantee that protocols are tested under the same conditions and we can observe the effect of densification on the performances of the methods. The 14 scenarios use 3 sink nodes corresponding to the 10th, 20th and 30th nodes. To facilitate the reproduction of these scenarios, readers can use the Microsoft Excel VBA source code presented in: https://cloud.femto-st.fr/nextcloud/index.php/s/KwGaNaQndyPC7zf and just set the number of nodes. The choice of the Microsoft Excel VBA environment is justified by the need for rapid prototyping, accessible and ease of use environment where the interrelationships with the other networking layers (MAC and modulation protocols) are momentarily ignored. We give in https://cloud.femtost.fr/nextcloud/index.php/s/YACPHYXHf5Fftqr the source file of including the simulator of the different compared methods.

For every scenario, we evaluate the average number of exchanged messages needed for one hundred randomly selected nodes (sources) to send their data to a sink as well as the corresponding success rate (number of times where the sent packet reaches at least one sink). We compared the results of M2MRP with the massive broadcast and SLR methods. Results of Table 2 show that M2MRP and SLR reduce significantly the number of exchanged messages compared to the naive broadcast approach. In M2MRP, the reduction of the number of sent messages is improved when the transmission angle is reduced $\left(60^{\circ}\right.$ in place of $\left.120^{\circ}\right)$. It should be mentioned that the number of sent messages in the flooding approach is equal to the number of nodes minus the number of sink nodes under the assumption that all nodes are reachable. The performance of M2MRP and SLR are equivalent on the square topology scenarios in terms of the number of sent messages. However, when the density of the network is low, M2MRP presents a better reliability. M2MRP protocol outperforms by far the SLR method on the bow tie topology scenarios. Indeed, whatever the density of the network is, the SLR approach fails to exceed $80 \%$ of success rate, while M2MRP succeeds $91 \%$ of times even with 100 nodes. The results of the Table 2 , show clearly the efficiency of our approach.

\section{CONCLUSION AND PERSPECTIVES}

In this paper we presented a new and efficient Multipoint-to-Multipoint Routing Protocol (M2MRP) for ultra-dense volatile nanonetworks. This protocol provides a powerful and scalable distributed procedure to dynamically and continuously compute the best transmission and reception directions of each node.

M2MRP protocol presents a natural way to express how the energy availability, congestion, radio quality and communication reliability should be taken into account for determining data routing paths over the network.

The simulation results are very encouraging and show great adaptability against network distribution and the comparison with SLR shows that M2MRP is clearly more efficient especially when the network topology presents non-convex parts.

The M2MRP protocol is implemented in AnyLogic Simulator and the source file can be found at:

https://cloud.femto-st.fr/nextcloud/index.php/s/34WEmRWa8KB22G3 However the achieved simulations merits closer examination in a multilayer network simulator. Our current works include the implementation of the M2MRP protocol over Network Simulator NS3.

Furthermore, more tests need to be conducted in order to assess the performance of the protocol under different volatility situations and with a more realistic modeling of energy consumption. In addition, the protocol can be improved to reduce even more the 


\begin{tabular}{|c|c|c|c|c|c|c|c|c|c|c|c|c|c|c|}
\hline & \multicolumn{7}{|c|}{ square topology } & \multicolumn{7}{|c|}{ bow tie topology } \\
\hline \#nodes & 100 & 200 & 300 & 400 & 500 & 750 & 1000 & 100 & 200 & 300 & 400 & 500 & 750 & 1000 \\
\hline \multirow[t]{2}{*}{ M2MRP(120o) } & 7,9 & 16,3 & 27,3 & 37,1 & 49,3 & 81,9 & 108,3 & 6,3 & 15 & 24,9 & 34,1 & 46,7 & 73,9 & 109,6 \\
\hline & $93 \%$ & $100 \%$ & $100 \%$ & $100 \%$ & $100 \%$ & $100 \%$ & $100 \%$ & $98 \%$ & $100 \%$ & $100 \%$ & $100 \%$ & $100 \%$ & $100 \%$ & $100 \%$ \\
\hline \multirow[t]{2}{*}{$\operatorname{M} 2 \mathrm{MRP}(60 \circ)$} & 5 & 8,1 & 15,2 & 18,1 & 24,2 & 38 & 50,9 & 3,7 & 7,2 & 11,7 & 15,4 & 19,4 & 30,1 & 44,2 \\
\hline & $76 \%$ & $97 \%$ & $99 \%$ & $99 \%$ & $100 \%$ & $100 \%$ & $100 \%$ & $91 \%$ & $94 \%$ & $94 \%$ & $96 \%$ & $96 \%$ & $96 \%$ & $96 \%$ \\
\hline \multirow[t]{2}{*}{ Flooding } & 95,1 & 193,1 & 294 & 393 & 497 & 739 & 997 & 94,1 & 195 & 291,1 & 393 & 497 & 724,6 & 987 \\
\hline & $100 \%$ & $100 \%$ & $100 \%$ & $100 \%$ & $100 \%$ & $100 \%$ & $100 \%$ & $100 \%$ & $100 \%$ & $100 \%$ & $100 \%$ & $100 \%$ & $100 \%$ & $100 \%$ \\
\hline \multirow[t]{2}{*}{ SLR } & 6,7 & 14,8 & 22,9 & 30,3 & 39,1 & 59,7 & 79,2 & 12,7 & 24,8 & 38,7 & 41,4 & 52 & 76,6 & 102 \\
\hline & $79 \%$ & $96 \%$ & $100 \%$ & $100 \%$ & $100 \%$ & $100 \%$ & $100 \%$ & $78 \%$ & $78 \%$ & $78 \%$ & $79 \%$ & $79 \%$ & $79 \%$ & $79 \%$ \\
\hline
\end{tabular}

Table 2: Complexity comparison of routing approaches according to the number of messages sent and the success rate.

amount of exchanged messages, for example by delaying the retransmission of received packets and wait to see if other nodes, that have received the packet, transmit it.

\section{REFERENCES}

[1] Hakim Mabed and Julien Bourgeois. Towards programmable material: Flexible distributed algorithm for modular robots shape-shifting. In 2014 IEEE/ASME International Conference on Advanced Intelligent Mechatronics, pages 408-414. IEEE, 2014.

[2] Vitaly Petrov, Dmitri Moltchanov, Maria Komar, Alexander Antonov, Pavel Kustarev, Shaloo Rakheja, and Yevgeni Koucheryavy. Terahertz band intrachip communications: Can wireless links scale modern x86 cpus? IEEE Access, 5:6095-6109, 2017.

[3] Vimal Upadhayay and Sonali Agarwal. Application of wireless nano sensor networks for wild lives. International fournal of Distributed and Parallel systems, 3(4):173, 2012.

[4] Rim Negra, Imen Jemili, and Abdelfettah Belghith. Wireless body area networks: Applications and technologies. In Procedia Computer Science, volume 83, 052016.

[5] Salah Abdulghani and M. F. M. Salleh. Overview of wireless mesh networks. fournal of Communications, 8:586-599, 092013.

[6] Thomas Heide Clausen, Philippe Jacquet, Cedric Adjih, Anis Laouiti, Pascale Minet, Paul Muhlethaler, Amir Qayyum, and Laurent Viennot. Optimized link state routing protocol (olsr). 012003.

[7] J. J. Garcia-Lunes-Aceves. Loop-free routing using diffusing computations IEEE/ACM Trans. Netw., 1(1):130-141, February 1993.

[8] Stefano; Imrich Chlamtac; Violet R. Syrotiuk; Barry A. Basagni. A distance routing effect algorithm for mobility (dream). In International Conference on Mobile Computing and Networking Proceedings of the 4th annual ACM/IEEE international conference on Mobile computing and networking, page 76-84. New York: ACM Press, 1998.

[9] Hemanth Narra, Yufei Cheng, Egemen Cetinkaya, Justin Rohrer, and James Sterbenz. Destination-sequenced distance vector (dsdv) routing protocol implementation in ns-3. 032011.
[10] Meeta Singh and Sudeep Kumar. A survey: Ad-hoc on demand distance vector (aodv) protocol. International fournal of Computer Applications, 161:38-44, 03 2017.

[11] Mehdi Kalantari and M Shayman. Routing in wireless ad hoc networks by analogy to electrostatic theory. pages 4028 - 4033 Vol.7, 072004.

[12] W Zhao, Z Tang, Y Yang, Y Wang, W Wang, and L Wang. Performance analysis of fluid dynamics model based routing for sensor networks. Fournal of Convergence Information Technology, 7:194-202, 032012.

[13] Ageliki Tsioliaridou, Christos Liaskos, Sotiris Ioannidis, and Andreas Pitsillides. Corona: A coordinate and routing system for nanonetworks. In Proceedings of the Second Annual International Conference on Nanoscale Computing and Communication, NANOCOM' 15, pages 1-6, New York, NY, USA, 2015. ACM.

[14] Angeliki Tsioliaridou, Christos Liaskos, Eugen Dedu, and Sotiris Ioannidis. Stateless linear-path routing for $3 \mathrm{~d}$ nanonetworks. In Proceedings of the $3 \mathrm{rd} \mathrm{ACM}$ International Conference on Nanoscale Computing and Communication, NANOCOM'16, pages 1-6, New York, NY, USA, 2016. ACM.

[15] Aydin Babakhani, Xiang Guan, Abbas Komijani, A. Natarajan, and A. Hajimiri. A 77-ghz phased-array transceiver with on-chip antennas in silicon: Receiver and antennas. IEEE Journal of Solid-State Circuits, 41:2795-2806, 2006.

[16] Ilker Demirkol, Cem Ersoy, and Ertan Onur. Wake-up receivers for wireless sensor networks: Benefits and challenges. Wireless Commun., 16(4):88-96, August 2009.

[17] Pietro Ciciriello, Luca Mottola, and Gian Pietro Picco. Efficient routing from multiple sources to multiple sinks in wireless sensor networks. In Proceedings of the 4th European Conference on Wireless Sensor Networks, EWSN'07, pages 34-50, Berlin, Heidelberg, 2007. Springer-Verlag.

[18] Kumar Viswanatha, Emrah Akyol, and Kenneth Rose. On optimum communication cost for joint compression and dispersive information routing. 2010 IEEE Information Theory Workshop, pages 1-5, 2010.

[19] Hadeel Elayan, Raed Shubair, and Asimina Kiourti. On graphene-based thz plasmonic nano-antennas. 112016.

[20] Veikko Lindroos, Markku Tilli, Ari Lehto, and T Motooka. Handbook of Silicon Based MEMS Materials and Technologies. 012010.

[21] Joan Capdevila, Josep Jornet, and Josep Solé-Pareta. Phlame: A physical layer aware mac protocol for electromagnetic nanonetworks. In IEEE Conference on Computer Communications Workshops, INFOCOM WKSHPS 2011, pages 431 - 436, 052011 